\title{
TUMOR DETECTION IN MR LIVER IMAGES BY INTEGRATING EDGE AND REGION INFORMATION *
}

\author{
Alexandra Branzan Albu ${ }^{1}$, Denis Laurendeau $^{1}$ And Christian Moisan ${ }^{2}$
}

\begin{abstract}
This paper describes a segmentation technique for 2D interventional MR images of liver tumours. Two features of MR data were likely to challenge existing segmentation methods. The first one is the inhomogeneous intratumoral texture, while the second one is the "blurred" appearance and the non-uniform sharpness of the tumour boundary. In order to detect the region of interest, we create the tumour contour map using a multithresholding technique and a measure of similarity between successive contours. Tumours presenting boundaries with non-uniform sharpness are segmented with an algorithm based on pixel aggregation and local textural information.
\end{abstract}

Résumé. Cet article décrit une méthode précise et fiable de segmentation pour des images RM des tumeurs de foie. Les approches traditionnelles de segmentation d'images ne donnent pas de bons résultats dans ce cas-là, à cause de deux caractéristiques particulières des données d'entrée : l'inhomogénéité de la texture intratumorale et l'apparence floue de la paroi des tumeurs. Pour détecter la région d'intérêt, nous créons une carte des contours de la tumeur en utilisant une technique de multi-seuillage et une mesure de similarité entre les contours successifs. Les tumeurs qui présentent des contours flous de contraste variable sont détectées avec un algorithme basé sur l'agrégation des pixels et sur l'information locale de texture.

\section{INTRODUCTION}

The segmentation process is essential for many applications using medical image data, such as quantification of morphological disease manifestations [1], identification of anatomical areas of interest for surgery planning paradigms or for radiation treatment [2], construction of anatomical models [3] etc.

Since only a few regions of a medical image provide useful information for further analysis, it is very important to extract these regions with high accuracy.

Our goal is to design and implement a segmentation tool adapted to 2D MR images of liver tumours. The region of interest (i.e. the tumour) is extracted from each segmented image of a sequence of 2D MR images corresponding to different anatomic slices and stored in a database. The resulting database will be further used for creating a 3D morphological model of the tumour.

We concentrate on two particular features of abdominal MRI data. The first one is the inhomogeneous texture of the liver tumours at a certain stage of their evolution. For instance, large-sized liver tumours develop a lobular appearance. The second feature is the non-uniform sharpness of the tumour boundary which may contain sharp

\footnotetext{
* This work was supported by NSERC, Grant no. 234773 under research project SKALPEL.

${ }^{1}$ Computer Vision and Systems Laboratory, Dept of Electrical and Computer Engineering, Laval University, Ste-Foy, Qc, Canada, G1K 7P4

2 iMRI Unit, Quebec City University Hospital, Qc, Canada, G1L 3L5
}

(c) EDP Sciences, SMAI 2003 
segments alternating with "blurred" segments. Due to infiltration into surrounding tissues, malignant liver lesions often present contours that do not reveal a clear-cut transition.

This paper describes our work toward a new segmentation method that allows a precise detection of the tumour region in MR liver images. The organization of the paper is as follows. Section 2 provides a brief overview of existing segmentation methods that were presented in the literature. Section 3 presents our original segmentation approach. Section 4 contains the experimental results obtained in order to validate the method and section 5 draws conclusions and describes future work.

\section{BACKGROUND}

The study of MR liver images is very poorly represented in the otherwise abundant literature on MR segmentation.

The interventional MR liver images are in general low-contrasted, containing tumours with ill-defined or "blurred" boundaries. The main cause of the "blurred" appearance is the movement artefact induced by respiration. While classical edge-detection methods do not produce reliable segmentation results, for MR images of the brain, active contours offer a very accurate detection of the region of interest [4]. Unfortunately, the use of active contours for the tumour detection in liver MR images is not suitable because of the "blurred" contours.

Texture analysis offers reliable statistical descriptors for region classification: cooccurrence matrices [5], measures of nonuniformity, homogeneity, contrast [6], etc. The statistical features are necessarily derived from a large number of pixels; hence they are not suited for pixel classification. A solution for this problem is offered by Markov random fields [7], a powerful mathematical tool that provides a stochastic textural model. A relaxation algorithm, as, for example, the ICM algorithm associated to the markovian model will converge towards the segmentation result [8].

In order to develop a robust segmentation technique for interventional MR liver images, we have not restricted our investigation to the MR segmentation literature. There are other types of medical images (mammography, computerized tomography) who may present challenges similar to our case. In [6], a computer-aided classification of benign and malignant masses on mammograms is attempted by computing gradient-based and texture-based features. Textural features are computed from grey-level cooccurrence matrices and are used to compare the textural information contained in mass regions and in mass margins. The gradient-based features are used to assess the sharpness of mass boundaries in a ribbon of pixels across mass margins.

A new segmentation approach presented in [9] is suitable for images containing objects with blurred boundaries. In our proposed segmentation approach, we use the concept of isolabel contour map defined in [11], but we develop a different strategy for building this contour map and a different measure of similarity between successive contours.

\section{Proposed Segmentation Approach}

Our approach aims towards an accurate segmentation of the tumour region in MR liver images. The database used for our study contains two different types of images. This classification is based on the appearance of the tumour, which is the region of interest in our case. The two different types of tumours are : a) tumour with smooth intrinsic texture and b) tumour with inhomogeneous intrinsic texture and boundaries of variable sharpness.

It is obvious that case a) is a rather simple segmentation problem, while case b) contains the real challenge of our study.

\subsection{Segmentation of smooth-textured liver tumours}

As in [9], we create an isolabel contour map in order to extract the boundaries of the region corresponding to the tumour. Our database contains only images with 256 grey levels (as in figure 1a). 


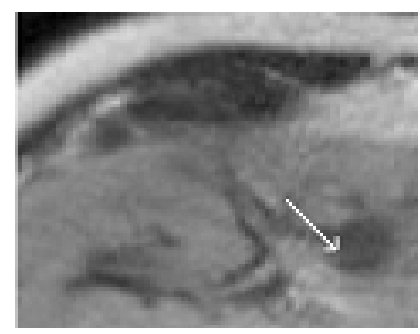

a)

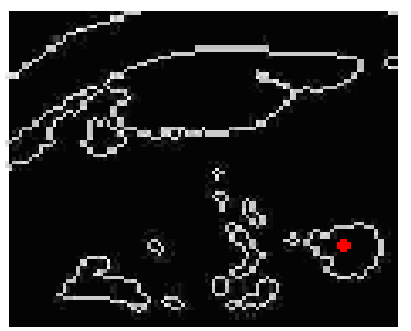

b)

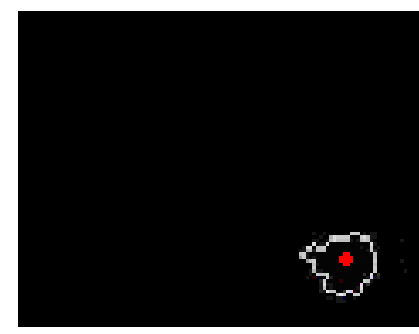

c)

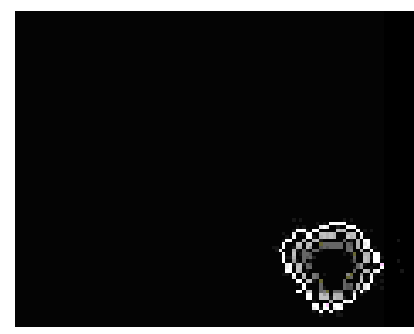

d)

FIGURE 1. a) the white arrow points at the tumour region belonging to a MR liver image ; b) isolabel contour $\mathrm{k}$ belonging to the image contour map; c) selection of the contour belonging to the tumour contour map; d) three similar contours belonging to the tumour contour map;

We first create the isolabel contour map of the entire image, then we select the contour map of the tumour, which is a subset of the image contour map.

To obtain the isolabel contour map, we iteratively threshold the grey level image 256 times. At each iteration, after thresholding, pixels belonging to the boundary between the two regions of the binarised image are labelled with the current threshold value. The set of pixels with an identical label forms an isolabel contour.

As a result of the iterative thresholding and boundary-extraction process, we obtain the isolabel contour map of the whole image, containing 256 isolabel contours. An instance of an isolable contour is shown in figure 1b).

In order to extract the isolabel contour map of the tumour, we need a minimum amount of information about its location in the liver. The radiologist is asked to select one single reference pixel located inside the tumour. We also need to know whether the targeted tumour is brighter or darker than the background. In T1-weighted MR images, tumours appear darker than the surrounding tissue while it is the opposite in T2-weighted MR images.

The extraction of the tumour isolabel contour map from the isolabel contour map of the image is an iterative process. The initial value of the label is set to the intensity value of the reference pixel. If the targeted tumour is brighter (darker) than the background, the label decreases (increases) one unit per iteration. We will further consider the tumour as the foreground of the image.

The central idea of the algorithm is that intensity in a bright (dark), relatively homogeneous foreground region gradually drops (rises) when approaching the boundary. When reaching the boundary, an abrupt intensity variation occurs. This transition depends on the local sharpness of the boundary. The transition from foreground to background generates a very different contour with respect to the contours enclosed in the foreground region. Since the tumour contour map contains only similar contours, the first dissimilar contour encountered marks the transition to the background and stops the generation process of the tumour contour map.

At every iteration, we select a subset of the analysed isolabel contour, which is a closed contour that encloses the reference pixel (see figure 1c). The reference pixel is marked in red in figures $1 \mathrm{~b}$ and $1 \mathrm{c}$.

Choosing this contour is done with successive morphological dilations of the reference pixel. The dilation process stops when reaching a closed contour having the label of the currently analysed isolabel contour. This contour is selected and further tested for inclusion/exclusion in/from the tumour isolabel contour map. If the selected contour is similar to the contour extracted at the previous iteration, it is included in the tumour contour map. If, on the contrary, it is dissimilar, it is excluded from the tumour contour map and the generation of the tumour contour map is complete. Figure $1 \mathrm{~d}$ ) shows three similar contours belonging to the tumour contour map. The boundary of the tumour is considered to be the last similar contour included in the tumour contour map.

In order to compare two contours, we propose a new measure of similarity. We consider two successive contours of the tumour contour map, $\operatorname{cont}(i)$ and $\operatorname{cont}(i-1)$ where the index represents the label value. If the targeted tumour is brighter than the background, cont $(i-1)$ always encloses cont $(i)$ for every possible value of $\mathrm{i}$, as in figure 3.3. Let $\operatorname{obj}(i)$ and $\operatorname{obj}(i-1)$ be the objects enclosed by $\operatorname{cont}(i)$ and by $\operatorname{cont}(i-1)$ respectively. We 


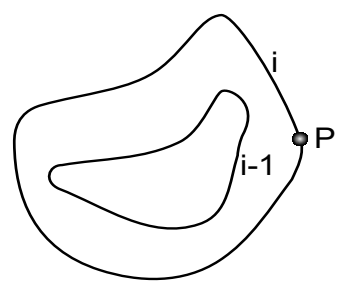

a)

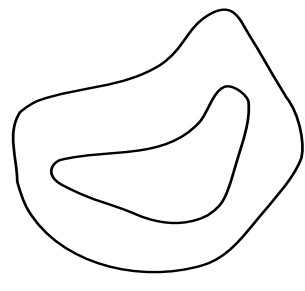

b)

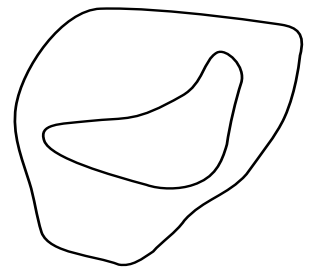

c)

Figure 2. a)morphological distance of P to obj(i); b)similar contours; c)dissimilar contours;

define a morphology-based distance between a pixel $\mathrm{P}$ in $\operatorname{cont}(i-1)$ and the object $\operatorname{obj}(i)$. Thus, the distance from $\mathrm{P}$ to $o b j(i)$ is equal to the minimum number of successive dilations of $\operatorname{obj}(i)$ performed in order to include $\mathrm{P}$ (see figure $2 \mathrm{a})$ ) :

$$
d(P, o b j(i))=\min \left\{n \mid n \in N, P \in \operatorname{obj}(i) \oplus K^{n}\right\}
$$

where the following structuring element is considered:

$$
K=\{(0,0),(0,1),(0,-1),(1,0),(-1,0)\}
$$

and $\oplus$ is the morphological dilation operator.

The distance between every pixel belonging to $\operatorname{cont}(i-1)$ and $\operatorname{obj}(i)$ is computed.

As a measure of similarity between $\operatorname{cont}(i-1)$ and $\operatorname{cont}(i)$ we use the variance :

$$
\sigma^{2}=\sum_{i=1}^{N}\left(d_{i}^{2}-\bar{d}^{2}\right) p\left(d_{i}\right)
$$

where $M$ is the number of points in contour cont(i-1), $p(d i)$ is the likelihood of distance di estimated from the distance histogram and $\bar{d}$ is the average distance between cont $(i-1)$ and $\operatorname{cont}(i)$ :

$$
\bar{d}=\sum_{i=1}^{N} d_{i} p\left(d_{i}\right)
$$

In order to decide whether cont $(i)$ and $\operatorname{cont}(i-1)$ are similar or dissimilar, we choose a threshold $\sigma_{0}^{2}$ for the variance $\sigma^{2}$. Thus, $\operatorname{cont}(i-1)$ and $\operatorname{cont}(i)$ are considered as dissimilar when $\sigma^{2}$ is greater than $\sigma_{0}^{2}$, and similar when $\sigma^{2}$ is less or equal to $\sigma_{0}^{2}$.

The proposed measure of similarity is sensitive to shape variations but not sensitive to size variations, as it is shown in figures $2 \mathrm{~b}$ ) and $2 \mathrm{c}$ ).

However, the proposed similarity measure is sensitive to the contour orientation. If two similar contours translate or rotate, their degree of similarity changes. This drawback is not relevant for our case, since the contours to be compared are successive contours of the tumour contour map and they are not allowed to translate or rotate.

\subsection{Segmentation of tumours with inhomogeneous texture and boundaries of variable sharpness}

The algorithm presented in paragraph 3.1 fails to detect the boundary of a contour with inhomogeneous texture and "blurred" boundaries. The last similar contour belonging to the tumour contour map is located inside the tumour (see figures $4 \mathrm{a}$ and $5 \mathrm{a}$ ). 

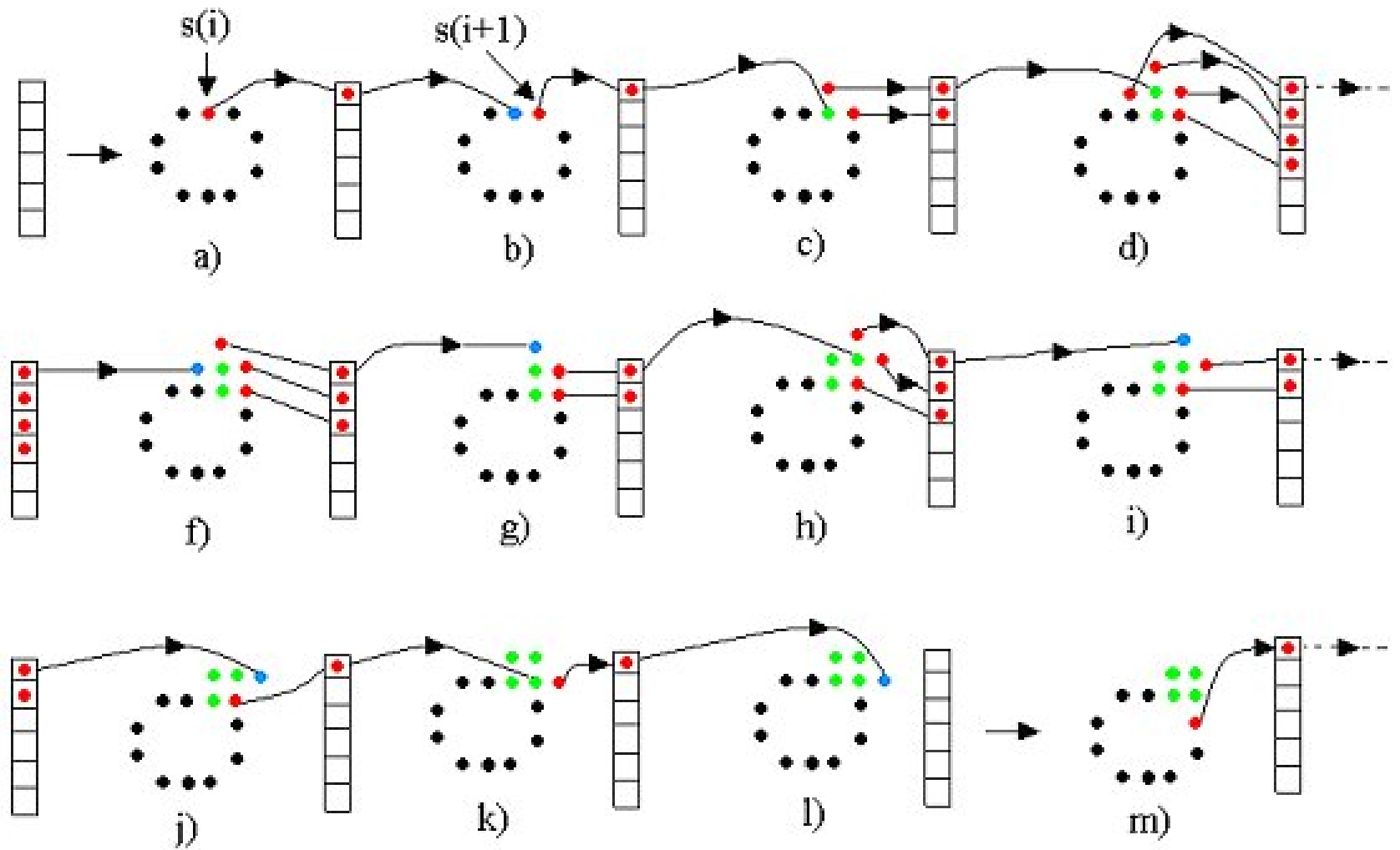

FiguRE 3. Two iterations of the segmentation algorithm based on pixel aggregation; pixels to be included in the LIFO queue are marked in red, pixels to be included in the grown region after evaluation are marked in green, while pixels to be excluded from the grown region are marked in blue.

To detect the real boundary of the tumour we propose a pixel aggregation process. A pixel aggregation algorithm must specify : a) the seed pixels and b) the local feature to be analysed for every candidate pixel for inclusion in the expanded region .

In our case, the seed pixels belong to the last similar contour of the tumour contour map. This initial contour is stored in a one-dimensional circular array. The trigonometric sense is used for moving around the contour.

The local feature to be analysed for every candidate pixel is texture-based and represents the correlation coefficient of two coocurrence matrices.

We compute the cooccurrence matrix $C$ of the object enclosed by the initial contour with the following relation:

$$
C(i, j)=\operatorname{card}\left\{\left(s, s^{\prime}\right) \mid f(s)=i, f\left(s^{\prime}\right)=j, s \in o b j_{0}, s^{\prime} \in o b j_{0}, s^{\prime} \in V_{4}(s)\right\}
$$

where $o b j_{0}$ is the object enclosed by the initial contour.

For every analysed pixel $s$ we consider a $11 \times 11$ patch, centered on the pixel and we compute its cooccurrence matrix $C B L O C(s)$. The size of the patch was chosen by considering the spatial limit of pixel interaction [10]. 
The correlation coefficient between $C B L O C(s)$ and $\mathrm{C}$ is computed with the following relation :

$$
R_{u v}=\frac{N \sum_{i=1}^{N} u_{i} v_{i}-\sum_{i=1}^{N} u_{i} \sum_{i=1}^{N} v_{i}}{\sqrt{\left(N \sum_{i=1}^{N} u_{i}^{2}-\left(\sum_{i=1}^{N} u_{i}\right)^{2}\right)\left(N \sum_{i=1}^{N} v_{i}^{2}-\left(\sum_{i=1}^{N} v_{i}\right)^{2}\right)}}
$$

where $\mathrm{u}, \mathrm{v}$ are two vectors with the same number $\mathrm{N}$ of elements.

Thus, for every candidate pixel s the texture-based local feature is :

$$
r(s)=R(C B L O C(s), C)
$$

This feature represents a measure for the interaction strength between pixel $s$ and the tumour region. Its range is limited to the interval $(0,1)$. A high value of $r(s)$ indicates that pixel $s$ strongly interacts with the region of interest and should be included in this region. On the contrary, a low value of $r(s)$ means that pixel $s$ should be excluded from the region. A threshold value $r_{0}$ for $r(s)$ is chosen, in order to decide the inclusion/exclusion of pixel $s$ from the tumour region.

The pixel aggregation process is an iterative algorithm. The number of iterations is equal to the number of seed pixels (i.e. the number of pixels belonging to the initial contour). The current seed pixel at iteration $i$ is the contour pixel $s(i)$. The current seed pixel is placed in an initially empty LIFO queue, which holds pixels to be evaluated for inclusion in the expanded region. The value $r(s(i))$ is compared to a predefined threshold $r_{0}$. If $r(s(i))$ is less than $r_{0}$, there is no pixel aggregation process for seed $s(i)$ and the next contour pixel $s(i+1)$ becomes the current seed pixel. If $r(s(i))$ is greater than $r_{0}$, the neighbors of $s(i)$ belonging to the current background are included in the LIFO queue. Furthermore, we extract pixel $p$ from the queue corresponding to the seed pixel $s(i)$. If $r(p)$ is greater than or equal to $r_{0}$, pixel $p$ is included in the expanded region and its neighbours belonging to the current background are included in the LIFO queue. If $r(p)$ is less than $r_{0}$, the next element of the LIFO queue is extracted and analysed. The region-growing process corresponding to the current seed pixel $s(i)$ stops when the LIFO queue is empty. Then, we move to the next seed pixel $s(i+1)$ and its corresponding pixel aggregation process begins.

Figure 3 shows two successive iterations $(i$ and $i+1$ ) of the segmentation algorithm based on pixel aggregation.

For every seed pixel $s(k)$ belonging to the initial contour, the algorithm grows a region $R(k)$. The final object corresponding to the tumour region is :

$$
o b j=o b j_{0} \bigcup\left(\bigcup_{i=1}^{N} R(k)\right)
$$

where $o b j_{0}$ is the object enclosed by the initial contour and $N$ is the total number of pixels belonging to the initial contour.

\section{Evaluation}

We evaluated the performance of our segmentation approach by comparing its results to those obtained by manual editing.

The manual segmentation was performed by a radiology-expert, who traced the contour of the tumour using conventional editing tools in a graphics interface.

We compared $S$, the object generated with our segmentation approach and $R$, the reference object enclosed by the manually edited contour. We considered the surface overlap metric [11], which performs a pair-wise comparison of two binary segmentations by relative overlap. Images are analysed pixel-by-pixel to calculate false positive, false negative, true positive and true negative pixels. 


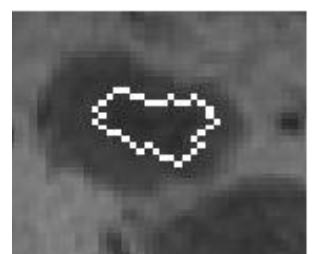

a)

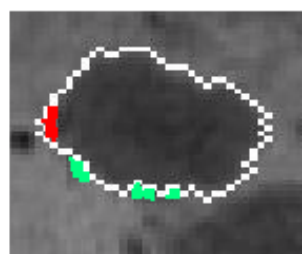

b)

FIGURE 4. a) Starting contour for segmentation of a smooth-textured tumour; b) Final segmentation result and evaluation.

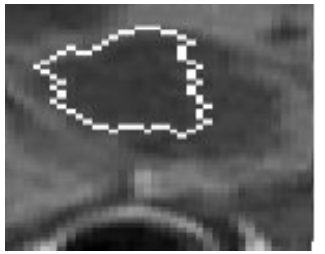

a)

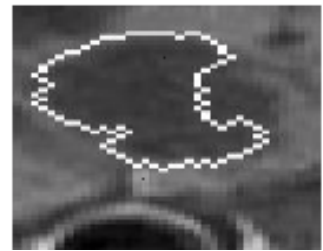

b)

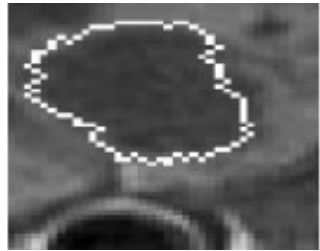

c)

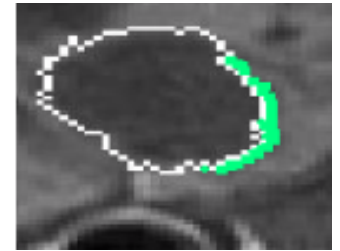

d)

FigURE 5. a) Starting contour for a tumour with inhomogeneous texture and fuzzy boundaries; b), c) Contours obtained respectively at second and fifth iteration of the segmentation algorithm; d) Final segmentation result and evaluation.

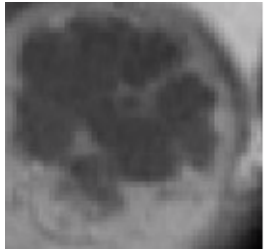

a)

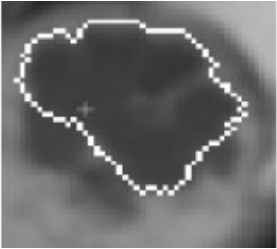

b)

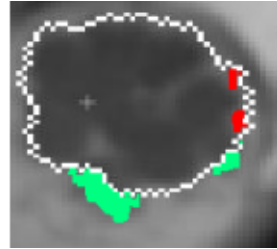

c)

FiguRE 6. a) The targeted tumour, with inhomogeneous texture and fuzzy boundaries; b) Starting contour; c) Final segmentation result and evaluation..

Let $N_{P}$ be the number of false positive pixels, $N_{N}$ the number of false negative pixels, $N_{R}$ the total number of reference pixels, and $N_{S}$ the total number of subject pixels.

We consider the following correspondence measure :

$$
C(S, R)=1-\frac{N_{N}+N_{P}}{N_{R}+N_{S}}
$$

with $N_{S}+N_{N}=N_{R}+N_{P}$. This measure gives a score of 1 if subject $S$ and reference $R$ are identical and 0 if they do not overlap.

Our database contains 10 patient data sets of MR interventional liver images, acquired at a magnetic intensity of 0.5 Tesla. No contrast agents were used in the acquisition process. The images were acquired on the General Electric Sigma Horizon open-field MR system at the Intervention Magnetic Resonance Imaging Unit of the Centre Hospitalier Universitaire de Québec (CHUQ). Reference segmentations were available for 15 images.

Figures 4, 5 and 6 show the initial stage, some intermediate instances and the final segmentation results of the segmentation algorithm for three liver tumours belonging to the database. False-positive and false-negative pixels are marked in red and green respectively. The threshold value for the contour similarity measure described 
TABLE 1. Quantitative results for the evaluation process.

\begin{tabular}{|c||c|c|c|c|c|}
\hline Tumour & $N_{S}$ & $N_{P}$ & $N_{N}$ & $N_{R}$ & $C(S, R) \%$ \\
\hline \hline $4 \mathrm{a}$ & 540 & 12 & 21 & 549 & 96.96 \\
\hline $5 \mathrm{a}$ & 604 & 0 & 56 & 660 & 95.56 \\
\hline $6 \mathrm{a}$ & 648 & 20 & 0 & 628 & 98.43 \\
\hline
\end{tabular}

in section 3.1 is set to $10 \%$, while the threshold value $r_{0}$ used in the pixel aggregation process is set to 0.25 . The quantitative results of the evaluation process for the three previously presented tumours are shown in table 1.

For the whole set containing 15 analysed images, the average value of the correspondence measure is $97.05 \%$.

\section{Conclusion}

Our segmentation approach takes into account the gradient, shape and texture information of Interventional MR images of liver tumours in order to detect accurately the targeted tumours.

The segmentation approach depends on the tumour type. We propose a simple segmentation approach for smooth-textured tumours. We use the concept of isolabel-contour maps [9], but introduce a new measure of similarity between successive contours. The boundary of the tumour is extracted by choosing the appropriate threshold value for the similarity measure.

The most challenging type of tumours are those with intrinsic texture and contours of variable sharpness. For this type, we propose a segmentation algorithm based on pixel aggregation.

The two presented approaches are not independent. Thus, the starting contour for the second approach is the last similar contour extracted from the tumour contour map. The approach based on pixel aggregation is used only when the previous approach does not provide satisfactory results.

The robustness of the proposed segmentation techniques is proven by the evaluation results.

Future work will concentrate on improving the robustness of the approach and on the development of an approach for selecting the best algorithm for tumour detection.

\section{REFERENCES}

[1] E. Chaney and S. Pizer, "Defining anatomical structures from medical images", Seminars Radiat. Oncol., vol. 2, pp.215-225, 1992.

[2] G. Tracton, E. Chaney, J. Rosenman, and S. Pizer, "Mask: Combining 2-D and 3-D segmentation methods to enhance functionality", Proc. SPIE Conf. Medical Imaging, Bellingham, WA, pp. 98-109,1994.

[3] J. F. Brinkley, "A flexible, generic model for anatomic shape: Application to interactive two-dimensional medical image segmentation and matching", Comput. Biomed. Res., vol. 26, no.2,pp. 121-142,1993.

[4] G. Hamarneh, T. McInerney, D. Terzopoulos, "Deformable Organisms for Automatic Medical Image Analysis", Proc. MICCAI Conf. Medical Image Computing and Computer-Assisted Intervention, Utrecht, pp. 66-76, 2001.

[5] P. A. Freeborough and N. C. Fox, "MR image texture analysis applied to the diagnosis and tracking of Alzheimer's disease", IEEE Trans. on Medical Imaging, vol.17, no.3, pp. 475-478, june 1995.

[6] N. R. Mudigonda, R. M. Rangayyan, J. E. Leo Desautels, "Gradient and Texture Analysis for the Classification of Mammographic Masses", IEEE Trans. on Medical Imaging, vol.19, no.10, pp. 1032-1043, oct 2000.

[7] M. Livin, N. Hanssen, P. Zerfass, E. Keeve, "3D Markov random fields and region growing for interactive segmentation of MR Data", Proc. MICCAI Conf. Medical Image Computing and Computer-Assisted Intervention, Utrecht, pp. 1191-1192, 2001.

[8] J. Besag, " Spatial interaction and the statistical analysis of lattice systems", J. of Royal Statistical Society, no. 2, pp.192-236, 1974.

[9] S. Shiffman, G. D. Rubin, S. Napel, "Medical Image Segmentation Using Analysis of Isolable-Contour Maps", IEEE Trans. on Medical Imaging, vol.19, no.11, pp. 1064-1074, nov 2000.

[10] F. Preteux, M. Moubarak, Ph. Grenier, "Pattern recognition in pulmonary computerized tomography images using Markovian modeling", SPIE/SPSE, San-Jose, CA , Proc. vol. 1224, feb 24-march1, 1991.

[11] G. Gerig, M. Jomier, M. Chakos, "Valmet: A new validation tool for assessing and improving 3D object segmentation", Proc. MICCAI Conf. Medical Image Computing and Computer-Assisted Intervention, Utrecht, pp. 516-523, 2001. 